\title{
Real-world outcomes in hereditary angioedema: first experience from the Icatibant Outcome Survey in the United Kingdom
}

\author{
Hilary J. Longhurst ${ }^{*}$, John Dempster ${ }^{2}$, Lorena Lorenzo ${ }^{2}$, Matthew Buckland ${ }^{2}$, Sofia Grigoriadou², \\ Christine Symons ${ }^{3}$, Claire Bethune ${ }^{3}$, Vincent Fabien ${ }^{4}$, Catherine Bangs ${ }^{5}$ and Tomaz Garcez ${ }^{5}$
}

\begin{abstract}
Background: Hereditary angioedema (HAE) is a potentially life-threatening, bradykinin-mediated disease, often misdiagnosed and under-treated, with long diagnostic delays. There are limited real-world data on best-practice management of HAE in the UK.

Objectives: To characterize the clinical profile, management and outcomes of patients with HAE type I and II from three specialist centres in the UK using data from the Icatibant Outcome Survey (IOS; Shire, Zug, Switzerland), an international observational study monitoring safety and effectiveness of icatibant, a selective bradykinin B2 receptor antagonist.

Methods: We performed retrospective analyses of IOS data for patients with HAE type I and II from three centres in the UK and compared UK data with pooled IOS data from 10 countries (48 centres).

Results: Analyses included 73 UK and 579 non-UK patients with HAE type I or II. Median diagnostic delay was 6.2 and 5.9 years, respectively. Analysis of data collected from February 2008 to July 2016 included 286 icatibant-treated attacks in 58 UK patients and 2553 icatibant-treated attacks in 436 non-UK patients (median of 3.0 attacks per patient in both groups). More attacks were treated by icatibant self-administration in UK patients (95.8\%) than in non-UK patients $(86.8 \%, \mathrm{p}<0.001)$. Time to icatibant treatment, time to resolution and attack duration were not significantly different in the UK versus non-UK patients.
\end{abstract}

Conclusion: UK patients from the specialist centres studied report similar diagnostic delay and similar icatibant treatment outcomes to their non-UK counterparts. However, improvements in the timely diagnosis of HAE are still required.

Trial registration ClinicalTrials.gov NCT01034969

Keywords: C1-inhibitor deficiency, Acquired angioedema, Hereditary angioedema, Icatibant, Icatibant Outcome Survey

\section{Background}

Hereditary angioedema (HAE) due to $\mathrm{C} 1$ inhibitor (C1-INH) deficiency is a rare autosomal dominant disease caused by mutations in the SERPING1 gene resulting in reduced levels (type I) or dysfunction (type II) of

\footnotetext{
*Correspondence: hlonghurst@doctors.org.uk

1 Department of Clinical Biochemistry and Immunology, Addenbrooke's

Hospital, Cambridge University Hospitals NHS Foundation Trust,

Cambridge, UK

Full list of author information is available at the end of the article
}

C1-INH. HAE type I and II attacks are characterized by recurrent swelling, commonly occurring in the skin, abdomen and larynx, which can be severe and debilitating $[1,2]$. In the UK, there are an estimated 1500 people with C1-INH deficiency, many of whom are undiagnosed [3]. A number of consensus international and UK guidelines regarding the diagnosis and management of $\mathrm{HAE}$ have been published [4-9]. However, the rarity of HAE means that non-specialist physicians are often unfamiliar 
with these conditions and patient management is usually conducted in coordination with tertiary centres, with particular emphasis on patient self-care [3, 9].

In the UK, intravenously administered plasma-derived C1-INH (Berinert ${ }^{\circledR}$, Cinryze $^{\circledR}$ ), recombinant C1-INH (Ruconest $^{\circledR}$ ) and the subcutaneously administered bradykinin receptor antagonist icatibant $\left(\right.$ Firazyr $\left.^{\circledR}\right)$ are licensed and effective for the treatment of acute attacks of HAE and are now commissioned nationally for homebased self-administration [10-15]. Following the initial approval of icatibant within Europe, the Icatibant Outcome Survey (IOS), a real-world patient registry documenting the clinical outcomes of patients treated with icatibant, was established to fulfil a European medicines agency post-marketing surveillance safety requirement. A number of clinical registries and surveys for HAE have been conducted in several countries [16-19], which may be used to support quality improvement activities. Although a recent UK national audit has helped to characterize the experience of patients with HAE [20], there is a relative paucity of UK data to help improve clinical practice and better understand the burden of disease.

This report summarizes observational UK data collected within the first 6 years of IOS (March 2010-July 2016) to characterize the clinical profile, management and outcomes of patients with HAE. These data could be used as an unofficial 'benchmark' of standards from three major centres within the UK that offer specialist management and up-to-date treatment protocols. In addition, data from the UK are compared with other IOS countries, with the aim of identifying potential areas for quality improvements.

\section{Methods}

\section{Study design and patients}

IOS (NCT01034969) is a prospective, international, observational study; study methodology has been published elsewhere [21]. The registry enrols patients with C1-INH deficiency (HAE types I and II), consistent with the European approval conditions for icatibant, but any patient prescribed subcutaneous icatibant treatment is eligible for inclusion. IOS is conducted in accordance with the Declaration of Helsinki and the international conference on harmonisation good clinical practice. Approval was obtained from ethics committees and/or local health authorities at all centres. Written, informed consent was obtained from all enrolled patients aged $\geq 18$ years ( $\geq 16$ years in the UK); consent was provided by parents/legal guardians for patients aged $<18$ years $(<16$ years in the UK).

The analyses described herein are based on IOS data collected between July 2009 and July 2016 for patients with HAE type I or II; patients with other forms of angioedema were excluded from this analysis. Retrospective data for attacks recorded prior to IOS enrolment were also collected, dating back to February 2008. During this period, a total of 51 centres in 11 countries contributed data for this analysis: Austria $(n=1)$, Brazil $(n=1)$, Germany $(n=7)$, Denmark $(n=1)$, Spain $(n=9)$, France $(n=17)$, Greece $(n=2)$, Israel $(n=4)$, Italy $(n=5)$, Sweden $(n=1)$ and the UK $(n=3)$. We also include a separate description of seven patients with acquired angioedema (AAE) due to C1-INH deficiency for this period from the UK sample, including one patient who was treated with icatibant for acute attacks (unlicensed indication).

Patient demographics and characteristics were recorded at enrolment (IOS entry [baseline]), including information on HAE attacks (including icatibant-treated) before IOS entry. Information relating to physical examination, icatibant treatment, concomitant medications and adverse events was collected at enrolment and at routine visits, recommended every 6 months thereafter. Data for attacks treated with icatibant or untreated attacks were recorded, including rescue medication and long-term prophylaxis usage; data for attacks treated with other products are not collected in IOS. For each icatibant-treated HAE attack, data were collected for time to treatment (time between the start of the attack and the first icatibant injection), duration of attack (time between onset of attack and complete resolution of symptoms) and time to resolution (time between first icatibant injection and complete resolution of symptoms) (Fig. 1). Data recorded for untreated attacks included frequency and duration of untreated attacks in the prior 12 months and potential reasons for non-treatment. The IOS protocol does not specify how patients should record attacks. Patients used several methods to record attacks, including diaries, evaluation forms and, in some cases, reporting attacks contemporaneously to specialist nurses. Self-administration of treatment included administration by family members or the patient, according to national prescribing information, after training in subcutaneous injection technique by a healthcare professional.

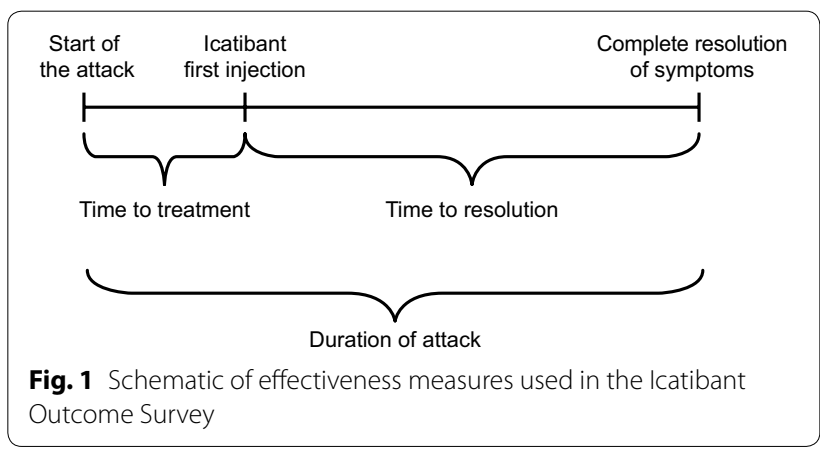


Attack severity was described by patients and was verified by a healthcare professional at routine visits. Severity was classified as: none (absence of symptoms); mild (mild interference with daily activities); moderate (moderate interference with daily activities and no other treatment required; severe (severe interference with daily activities and with or without other treatment); and very severe (very severe interference with daily activities and other treatment required) [22].

\section{Statistical analyses}

Results of UK versus non-UK populations were compared. A mixed-model analysis of repeated measures (Proc Mixed; SAS Institute, Cary, NC, USA) was used to compare time to treatment, time to resolution and duration of attack. The Chi squared test was used for the comparison of dichotomous data, with a statistical significance level of alpha $=0.05$. Data are presented as median (interquartile range [IQR]) or mean (standard deviation $[\mathrm{SD}])$, unless otherwise specified.

\section{Results}

\section{Patient characteristics}

Between July 2009 and July 2016, 652 patients diagnosed with HAE type I or II were entered into the IOS database. Of these, 73 patients were from three centres in the UK (enrolled from March 2010 onwards) and 579 patients were from 48 centres outside the UK. Approximately $60 \%$ of patients in both UK and non-UK groups were female. Median patient age at extract was 42.2 and 43.3 years for UK and non-UK patients, respectively. Patient demographics and baseline characteristics are presented in Table 1. In the UK, one patient received icatibant under the age of 18 years (two icatibant injections, one each for two separate attacks at 17.6 and 17.8 years). Outside the UK, five patients received icatibant injections under the age of 18 years (range 16.2-17.9 years). Of these five nonUK patients, two patients each received a single icatibant injection, one patient received three icatibant injections for three separate attacks, one patient received four icatibant injections for four separate attacks and one patient received ten icatibant injections for ten separate attacks.

\section{Socioeconomic data}

Employment status for UK and non-UK patients at IOS entry is shown in Table 1. In the UK, a significantly higher proportion of patients were employed or self-employed compared with non-UK patients; 53/73 (72.6\%) versus $233 / 419$ (55.6\%); $\mathrm{p}=0.0111$. The proportion of students among the UK and non-UK patients was similar: 8/73 (11.0\%) and 53/419 (12.6\%), respectively. More patients in the UK missed work or education prior to IOS compared with non-UK patients $(63.3 \%$ versus
Table 1 Patient demographics and baseline characteristics

\begin{tabular}{|c|c|c|}
\hline \multirow[t]{2}{*}{ Characteristic } & \multicolumn{2}{|c|}{ Patients with HAE type I or II } \\
\hline & $\begin{array}{l}\text { UK } \\
(N=73)\end{array}$ & $\begin{array}{l}\text { Non-UK } \\
(N=579)\end{array}$ \\
\hline \multicolumn{3}{|l|}{ Age at extract, years } \\
\hline Mean (SD) & $42.9(14.7)^{\mathrm{a}}$ & $45.1(15.2)^{b}$ \\
\hline Median (range) & $42.2(18-82)^{a}$ & $43.3(6-86)^{b}$ \\
\hline \multicolumn{3}{|l|}{ Gender, $n(\%)$} \\
\hline Male & $29(39.7)$ & $235(40.6)$ \\
\hline Female & $44(60.3)$ & $344(59.4)$ \\
\hline \multicolumn{3}{|l|}{ Age at first symptoms, years } \\
\hline Mean (SD) & $11.3(9.5)^{c}$ & $14.1(11.2)^{d}$ \\
\hline Median (IQR) & $10.0(5.0,16.0)^{c}$ & $13.0(6.0,19.0)^{d}$ \\
\hline \multicolumn{3}{|l|}{ Age at diagnosis, years } \\
\hline Mean (SD) & $21.5(12.7)^{\mathrm{e}}$ & $24.6(15.9)^{f}$ \\
\hline Median (IQR) & $18.9(12.6,30.0)^{\mathrm{e}}$ & $21.0(13.2,34.2)^{f}$ \\
\hline \multicolumn{3}{|c|}{ Delay between first symptoms and diagnosis, years } \\
\hline Mean (SD) & $9.5(13.9)^{c}$ & $10.5(13.6)^{9}$ \\
\hline Median (IQR) & $6.2(0.0,17.5)^{c}$ & $5.9(0.4,17.6)^{9}$ \\
\hline \multicolumn{3}{|l|}{ Employment status, $n(\%)^{\mathrm{h}}$} \\
\hline Employee & $46(63.0)$ & $218(52.0)$ \\
\hline Self-employed & $7(9.6)$ & $15(3.6)$ \\
\hline Homemaker & $3(4.1)$ & $20(4.8)$ \\
\hline Leave of absence/sabbatical & $1(1.4)$ & $2(0.5)$ \\
\hline Pre-school & 0 & $4(1.0)$ \\
\hline Retired & $3(4.1)$ & $42(10.0)$ \\
\hline Student & $8(11.0)$ & $53(12.6)$ \\
\hline Unemployed & $4(5.5)$ & $34(8.1)$ \\
\hline Other/unknown & $4(5.5)$ & $43(10.3)$ \\
\hline
\end{tabular}

HAE hereditary angioedema, IQR interquartile range, $S D$ standard deviation

a $n=73$

b $n=579$

c $n=58$

d $n=497$

e $n=70$

${ }^{\mathrm{f}} n=535$

${ }^{9} n=486$

h Patients could have more than one employment status (UK sample $n=73$, non-UK patients $n=419$ )

$37.6 \%$, respectively; $\mathrm{p}=0.009)$ and during the IOS observation period $(54.9 \%$ versus $24.9 \%$ respectively, $\mathrm{p}<0.001)$ (Fig. 2). The proportion of patients missing days of work or education improved in the non-UK patients during the IOS observation period (decreasing from 37.6 to $24.9 \%$ ) The proportion of patients missing days of work or education showed a non-significant trend to improvement in the UK during the IOS observation period (decreasing from 63.3 to $54.9 \%, \mathrm{p}=0.060$ ); however the reduction was seen only in patients reporting absences of $\leq 7$ days. More patients in the UK required hospitalisation prior 


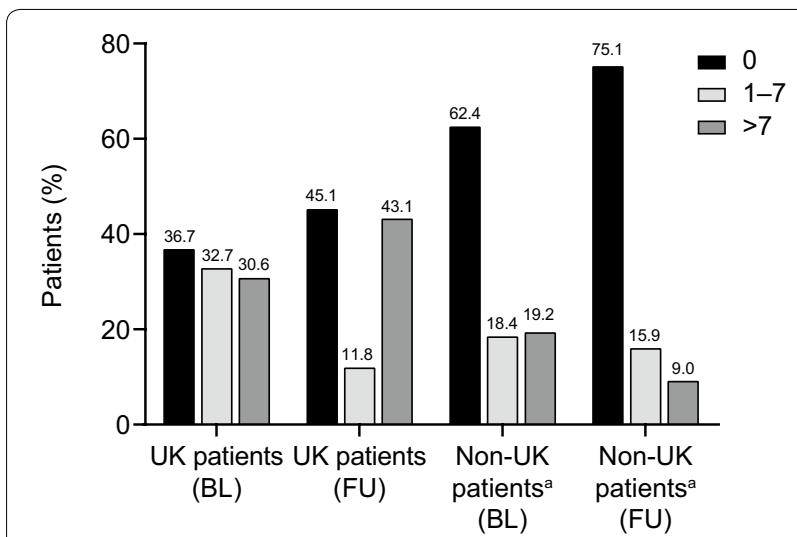

Number of missed day(s) of work/education

Fig. 2 Proportion of the UK and non-UK IOS patients with days of missed work or education prior to IOS entry and during the IOS observation period. For the UK and non-UK patients, respectively, $n=49$ and $n=125$ for the period before IOS entry and $n=51$ and $n=201$ for the IOS follow-up period. BL baseline (12 months prior to IOS entry), FU follow-up, IOS Icatibant Outcome Survey. ${ }^{a}$ Non-UK countries are Austria, Brazil, Denmark, France, Germany, Greece, Israel, Italy, Spain and Sweden

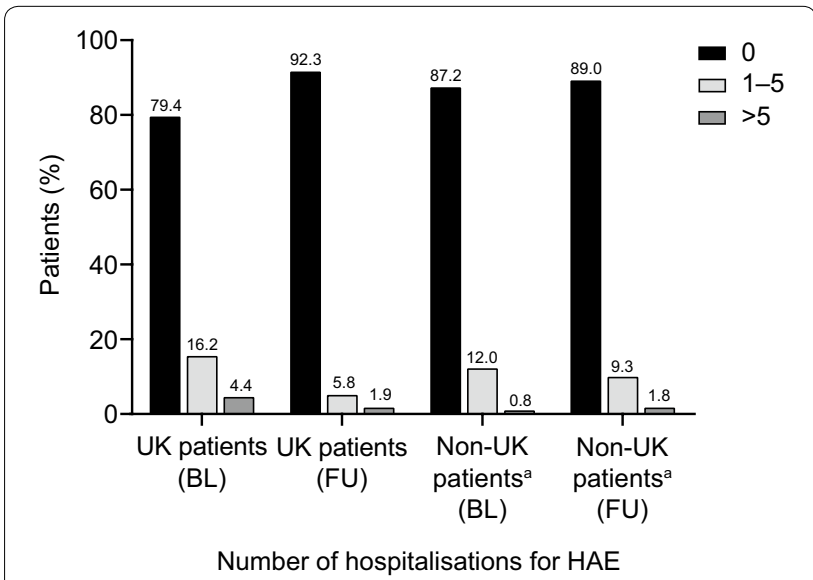

Fig. 3 Proportion of the UK and non-UK IOS patients hospitalized at baseline (prior to IOS entry) and during IOS observation. For the UK and non-UK patients, respectively, $n=68$ and $n=367$ for the period before IOS entry and $n=52$ and $n=399$ for the IOS follow-up period. BL baseline (12 months prior to IOS entry), FU follow-up, IOS Icatibant Outcome Survey. ${ }^{a}$ Non-UK countries are Austria, Brazil, Denmark, France, Germany, Greece, Israel, Italy, Spain and Sweden

to IOS compared with non-UK patients $(20.6 \%$ versus $12.8 \%$, respectively; $\mathrm{p}=0.037$ ), however no difference in rates of hospitalisation during the IOS observation period between UK and non-UK patients was observed $(p=0.696)$ (Fig. 3). However, within the UK the rate of hospitalisations prior to IOS compared to the IOS observation period showed a trend to improvement but this did not reach statistical significance $(\mathrm{p}=0.098)$.

\section{Use of long-term prophylaxis}

In UK and non-UK patients, respectively, ongoing longterm prophylaxis use at IOS entry and during the follow-up period was $75.3 \%$ (55/73) and 44.4\% (257/579). Attenuated androgens (danazol, stanozolol and oxandrolone) were the most commonly used ongoing longterm prophylaxis medication (at IOS entry and during the follow-up period) by both UK and non-UK patients (accounting for 59.9 and $66.9 \%$ of usage, respectively). In the UK, attenuated androgens were used as ongoing long-term prophylaxis in $54.5 \%(18 / 33)$ of patients from the Barts Health NHS Trust, London; 71.4\% (10/14) of patients from the Central Manchester University Hospital NHS Foundation Trust, Manchester and 62.5\% (5/8) of patients from Plymouth Hospital NHS Trust. Other ongoing long-term prophylactic agents used in the UK overall were C1-INH $(7.3 \% ; 4 / 55)$ and tranexamic acid $(23.6 \%, 13 / 55)$.

\section{Delay in diagnosis}

Median age at diagnosis of HAE type I or II was 18.9 years for the UK patients and 21.0 years for non-UK patients, with a median delay between first symptoms and diagnosis of 6.2 and 5.9 years, respectively (Table 1 ). Of 58 patients in the UK with available data, $36(62.1 \%)$ patients experienced a delay in diagnosis of $\geq 2$ years, with $23(39.7 \%)$ patients having a delay of $\geq 10$ years (Fig. 4). There was a clear biphasic distribution in time from symptoms to diagnosis.

\section{Treatment of HAE attacks with icatibant Attack rate}

Among 73 patients in the UK, 58 (79.5\%) had reported at least one attack treated with icatibant, with a total of 286 icatibant-treated attacks. These attacks were derived

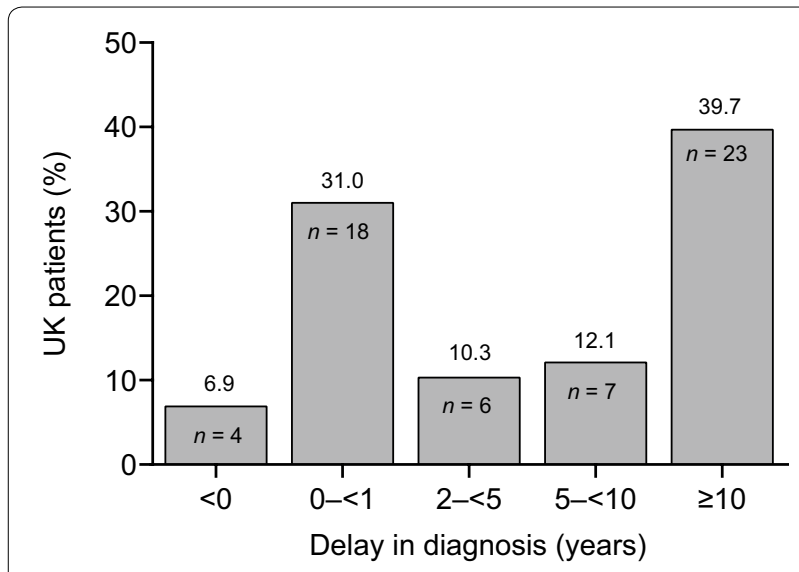

Fig. 4 Proportion of HAE patients in the UK sample with a delay in diagnosis. HAE hereditary angioedema. $n=58$ 
Table 2 HAE type I or II attacks treated with icatibant in the UK sample

\begin{tabular}{|c|c|c|c|c|c|c|c|c|}
\hline \multirow[t]{2}{*}{ IOS period } & \multicolumn{2}{|c|}{$\begin{array}{l}\text { Barts health NHS trust, } \\
\text { London } \\
N=31\end{array}$} & \multicolumn{2}{|c|}{$\begin{array}{l}\text { Central Manchester univer- } \\
\text { sity hospital NHS foundation } \\
\text { trust, Manchester } \\
N=16\end{array}$} & \multicolumn{2}{|c|}{$\begin{array}{l}\text { Plymouth hospitals NHS } \\
\text { trust } \\
N=11\end{array}$} & \multicolumn{2}{|c|}{$\begin{array}{l}\text { All UK sample } \\
N=58\end{array}$} \\
\hline & Patients, $n$ & Attacks, $n$ & Patients, $n$ & Attacks, $n$ & Patients, $n$ & Attacks, $n$ & Patients, $n$ & Attacks, $n$ \\
\hline Before IOS entry & 23 & 40 & 11 & 84 & 11 & 21 & 45 & 145 \\
\hline After IOS entry & 24 & 146 & 12 & 124 & 5 & 20 & 41 & 290 \\
\hline Total & 31 & 186 & 16 & 208 & 11 & 41 & 58 & 435 \\
\hline
\end{tabular}

HAE hereditary angioedema, IOS Icatibant Outcome Survey

Table 3 Comparison of HAE attacks treated with icatibant and untreated attacks

\begin{tabular}{|c|c|c|}
\hline \multirow[t]{2}{*}{ Parameter } & \multicolumn{2}{|c|}{$\begin{array}{l}\text { Patients with HAE type } \\
\text { I or II }\end{array}$} \\
\hline & $\begin{array}{l}\text { UK } \\
N=73\end{array}$ & $\begin{array}{l}\text { Non-UK } \\
N=579\end{array}$ \\
\hline \multicolumn{3}{|l|}{ Icatibant-treated attacks ${ }^{\mathrm{a}}$} \\
\hline Patients treated with icatibant, $n$ & 58 & 436 \\
\hline \multicolumn{3}{|l|}{ Attacks treated with icatibant per patient } \\
\hline Mean (SD) & $7.5(12.8)$ & $7.6(12.5)$ \\
\hline Median (IQR) & $3.0(1.0,6.0)$ & $3.0(1.0,8.0)$ \\
\hline \multicolumn{3}{|l|}{ Untreated attacks in year prior to IOS entry ${ }^{b}$} \\
\hline \multicolumn{3}{|l|}{ Untreated attacks per patient } \\
\hline Mean (SD) & $8.5(16.8)$ & $7.4(14.0)$ \\
\hline Median (IQR) & $2.0(0,5.0)$ & $2.0(0,8.0)$ \\
\hline \multicolumn{3}{|l|}{ Untreated attacks in the IOS observation period ${ }^{a}$} \\
\hline Patients with at least one untreated attack, $n$ & $30^{e}$ & $198^{f}$ \\
\hline \multicolumn{3}{|l|}{ Untreated attacks per patient } \\
\hline Mean (SD) & $10.9(23.2)$ & $8.2(17.3)$ \\
\hline Median (IQR) & $2.0(0.0,8.0)$ & $1.0(0,8.0)$ \\
\hline \multicolumn{3}{|c|}{$\begin{array}{l}\text { HAE hereditary angioedema, IOS Icatibant Outcome Survey, IQR interquartile } \\
\text { range, } S D \text { standard deviation }\end{array}$} \\
\hline \multicolumn{3}{|c|}{ a Attacks in year prior to IOS entry and through the IOS observation period } \\
\hline \multicolumn{3}{|c|}{$\begin{array}{l}{ }^{\mathrm{b}} \text { Untreated attacks were defined as attacks not treated with icatibant or any } \\
\text { other treatment }\end{array}$} \\
\hline \multicolumn{3}{|l|}{ c 19 patients had no untreated attacks } \\
\hline \multicolumn{3}{|l|}{ d 114 patients had no untreated attacks } \\
\hline \multicolumn{3}{|l|}{ e 22 patients had no untreated attacks } \\
\hline f 150 patients had no untreated attacks & & \\
\hline
\end{tabular}

from 31 patients from the Barts Health NHS Trust, London, 16 patients from the Central Manchester University Hospital NHS Foundation Trust, Manchester and 11 patients from the Plymouth Hospitals NHS Trust, Plymouth (Table 2). A total of 2553 icatibant-treated attacks were reported for 436/579 (75.3\%) patients in countries outside the UK. A median of 3.0 attacks per patient were treated with icatibant in both the UK and outside the UK (Table 3).

\section{Icatibant-treated versus untreated attacks}

In total, 854 attacks were recorded after IOS entry for the 73 UK patients. Of these, 286 (33.5\%) attacks were treated with icatibant and 568 (66.5\%) remained untreated. As IOS is an icatibant registry, data for attacks treated with C1-INH were not recorded. For non-UK patients, 5423 attacks were recorded after IOS entry, with 2553 (47.1\%) attacks treated with icatibant and $2870(52.9 \%)$ attacks not treated with any treatment. In UK patients, the median number of untreated attacks per patient was the same (2.0) in the IOS observation period (data collected at routine visits) as in the period prior to IOS entry (data collected at baseline) (Table 3). For non-UK patients, the median number of untreated attacks per patient was lower (1.0 versus 2.0, respectively) in the IOS observation period than in the period prior to IOS entry. Since C1-INH-treated attacks were not recorded, no conclusion can be drawn as to overall percentages. However, it remains clear that a substantial numbers of attacks are untreated and that in the UK, these outnumber those treated with icatibant by almost 2-1. Although we do not have data on severity or complete data on possible reasons for non-treatment, the duration of untreated attacks, both prior to IOS enrolment (median $72 \mathrm{~h}$ for both UK and non-UK patients) and IOS follow-up period (72 versus $79 \mathrm{~h}$ for the UK and non-UK patients, respectively) is substantially longer than for treated attacks $(9.0$ versus $8.6 \mathrm{~h}$; Table 4).

\section{Severity of attacks}

In the UK, $65.5 \%(279 / 426)$ of attacks before treatment were classified (where data were available) as severe/very severe; $26.1 \%(111 / 426)$ as moderate; and $8.5 \%(36 / 426)$ as mild or very mild. For non-UK patients, attack severity before treatment was classified as severe/very severe, 53.3\% (1441/2704 attacks); moderate, 37.2\% (1005/2704 attacks); and mild or very mild, 9.5\% (258/2704 attacks). No difference in attack severity was observed between UK and non-UK patients $(\mathrm{p}=0.1724)$. 
Table 4 Time to treatment, time to resolution and duration of icatibant-treated HAE attacks

\begin{tabular}{|c|c|c|c|c|c|c|c|}
\hline \multirow[t]{3}{*}{ Endpoint } & \multicolumn{7}{|c|}{ Patients with HAE type I or II } \\
\hline & \multicolumn{3}{|c|}{$\begin{array}{l}\text { UK } \\
(N=73)\end{array}$} & \multicolumn{3}{|c|}{$\begin{array}{l}\text { Non-UK } \\
(N=579)\end{array}$} & \multirow[t]{2}{*}{$p$ value ${ }^{b}$} \\
\hline & $n^{\mathrm{a}}$ & Mean (SD) & Median (IQR) & $n^{\mathrm{a}}$ & Mean (SD) & Median (IQR) & \\
\hline Time from attack onset to treatment, $h^{c}$ & 222 & $2.9(4.8)$ & $0.8(0.4,3.0)$ & 1120 & $3.9(7.3)$ & $1.3(0.5,4.0)$ & 0.0632 \\
\hline Time to complete symptom resolution, $\mathrm{h}^{\mathrm{d}}$ & 222 & $10.5(14.1)$ & $6.0(1.3,14.0)$ & 1120 & $11.8(16.1)$ & $5.8(2.0,14.1)$ & 0.2774 \\
\hline Duration of attack, $h^{e}$ & 222 & $13.4(15.4)$ & $9.0(2.5,18.5)$ & 1120 & $15.6(18.9)$ & $8.6(4.0,20.0)$ & 0.1022 \\
\hline \multicolumn{8}{|c|}{ HAE hereditary angioedema, IQR interquartile range, $n$ number of evaluable attacks, $S D$ standard deviation } \\
\hline \multicolumn{8}{|c|}{ attacks with complete data for time to treatment, time to complete resolution and attack duration, excluding attacks treated $>100 \mathrm{~h}$ after attack onset } \\
\hline \multicolumn{8}{|c|}{ b Mixed-model analysis of repeated measures comparing the UK versus non-UK IOS datasets } \\
\hline \multicolumn{8}{|c|}{ c Time between the start of the attack and the first icatibant injection } \\
\hline \multicolumn{8}{|c|}{${ }^{d}$ Time between first injection of icatibant and complete resolution of symptoms } \\
\hline \multicolumn{8}{|c|}{ e Time between start of attack and complete resolution of symptoms } \\
\hline
\end{tabular}

\section{Use of icatibant self-administration}

Of the attacks treated with icatibant, the majority were treated by self-administration both inside and outside the UK (95.8\% versus $86.8 \%$, respectively) (Fig. 5) with the rate of self-administration in the UK significantly higher $(\mathrm{p}<0.001)$ compared to non-UK patients. Healthcare professionals administered icatibant for first attacks in around one-third of UK and non-UK patients in the IOS observation period (16/57 [28.1\%] and 133/366 [36.3\%], respectively). The mode of icatibant administration for the first five attacks for UK patients is shown in Fig. 6.

\section{Time to icatibant treatment, time to resolution and attack duration}

Time to treatment with icatibant, time to resolution and duration of attack were not significantly different

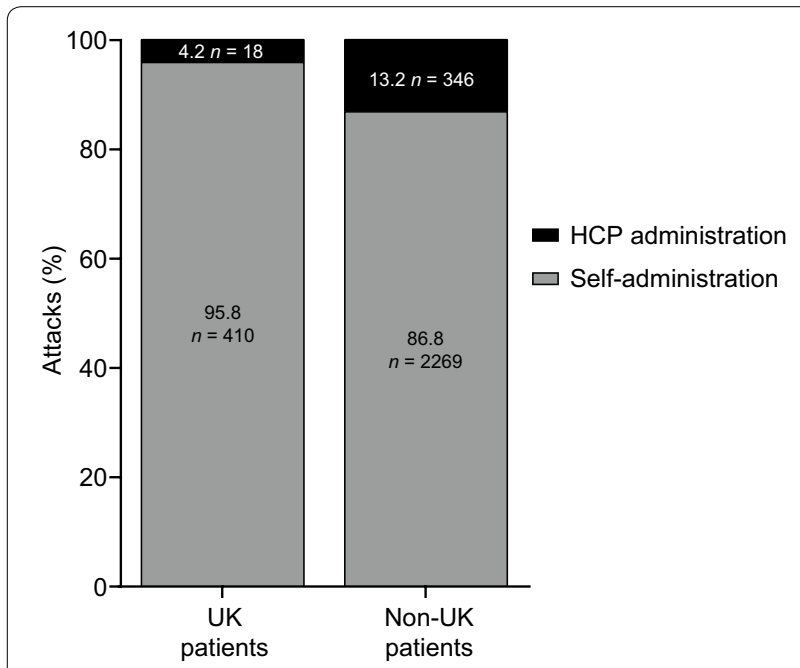

Fig. 5 Proportion of HAE attacks treated with icatibant by self-administration or administration by HCPs per attack in the UK and non-UK patients. HAE hereditary angioedema, HCP healthcare professional in the UK compared with non-UK patients, although there was a trend to earlier treatment in UK patients, $\mathrm{p}=0.0632, \mathrm{p}=0.2774$ and $\mathrm{p}=0.1022$, respectively (Table 4, Fig. 7).

\section{Use of $\mathrm{C1}$-INH rescue}

In the UK, excluding one outlier with a very atypical pattern of use (described below), C1-INH was used as rescue medication in $12.7 \%$ of icatibant-treated attacks (48/378 attacks in $15 / 57$ patients) compared with $9.2 \%$ of icatibant-treated attacks (304/3306 attacks in 86/436 patients) in non-UK patients.

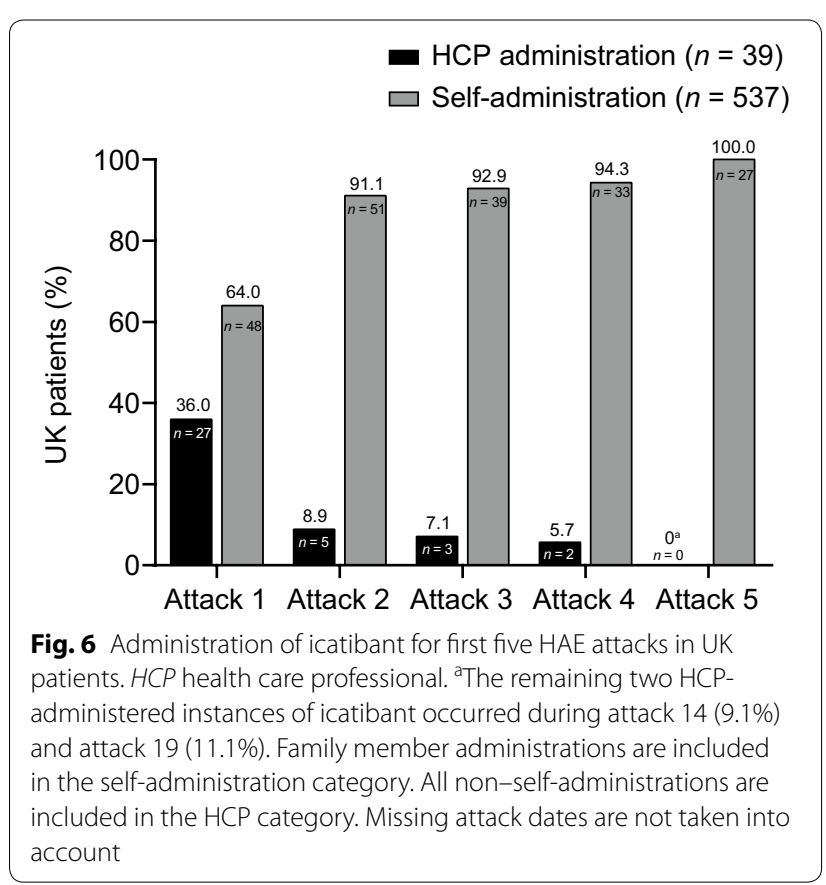



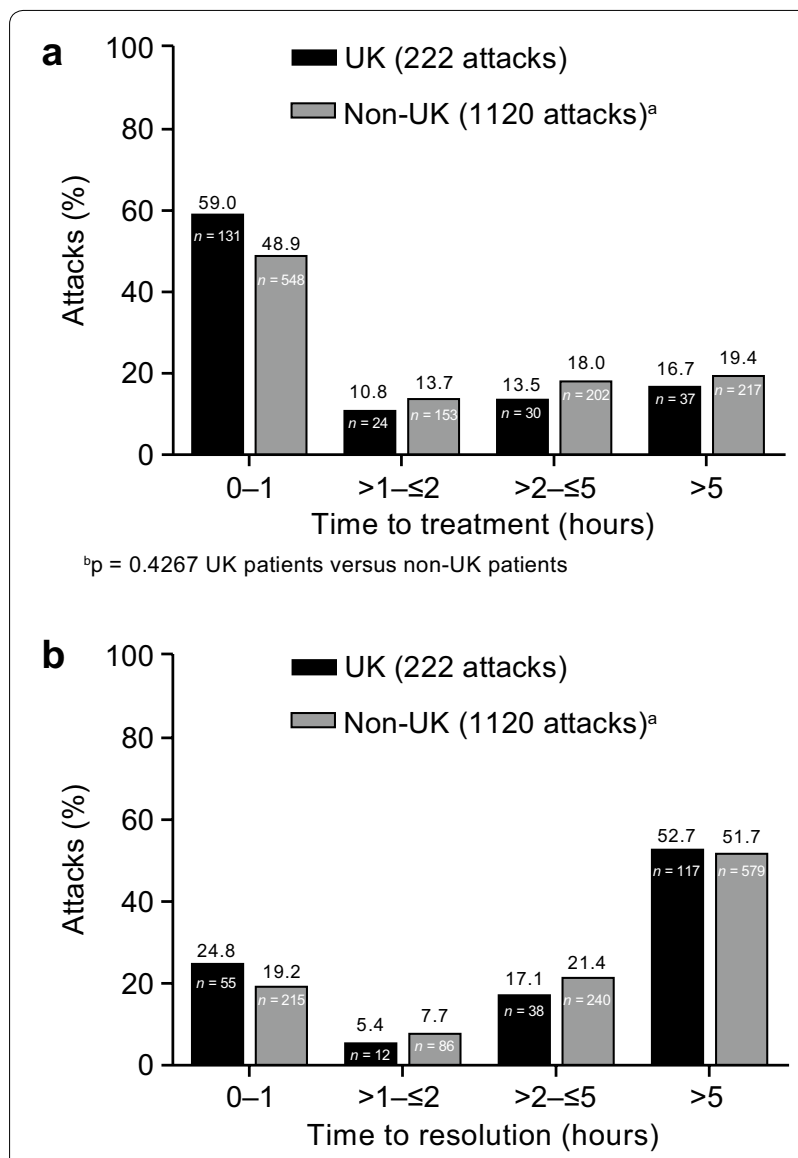

${ }^{b} p=0.8440$ UK patients versus non-UK patients

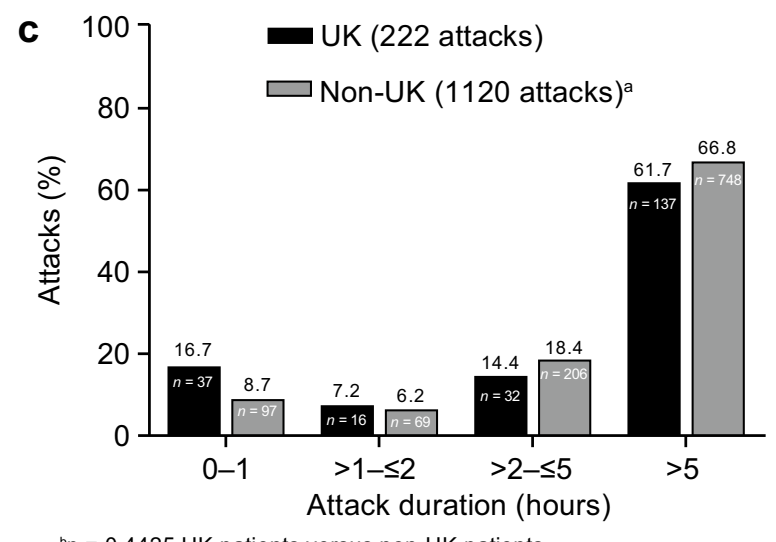

${ }^{b} p=0.4425$ UK patients versus non-UK patients

Fig. 7 Proportion of icatibant-treated HAE attacks (with complete data for time to treatment, time to resolution and attack duration) in UK patients and non-UK patients according to $\mathbf{a}$ time to treatment, $\mathbf{b}$ time to symptom resolution and $\mathbf{c}$ duration of attack. HAE hereditary angioedema, Time to treatment time between the start of the attack and the first icatibant injection, Time to resolution time between first injection of icatibant and complete resolution of symptoms, Attack duration time between start of attack and complete resolution of symptoms. ${ }^{2}$ Non-UK countries are Austria, Brazil, Denmark, France, Germany, Greece, Israel, Italy, Spain and Sweden. ${ }^{\circ}$ Generalized linear model for repeated measures

\section{Clinical experience: patient with high icatibant reinjection} and $\mathrm{C} 1-\mathrm{INH}$ rescue medication usage

Further analysis of the pattern of icatibant usage revealed that one patient in the UK had a high rate of icatibant reinjection combined with high usage of $\mathrm{C} 1-\mathrm{INH}$ rescue medication. This 50-year-old man with HAE type I selfadministered icatibant for 57 HAE attacks (very severe, $n=12$; severe, $n=39$; and moderate, $n=6$ ) between 22 October 2009 and 25 October 2014. Approximately half $(27 / 57)$ of these attacks were treated with a second injection of icatibant and $86.0 \%(49 / 57)$ with C1-INH rescue medication. The median (IQR) time to first icatibant injection was $0.5(0.5-1.0) \mathrm{h}$, median (IQR) time to complete resolution was $1.0(0.5-9.5) \mathrm{h}$ and median (IQR) duration of attack was $3.5(1.0-11.0)$ h. Median (IQR) time between first and second icatibant injection was $9.0(6.0-12.0) \mathrm{h}$ and the median (IQR) time between second injection and complete resolution was $0.5(0.5-$ 0.5 ) h. During the treatment period the patient was under intense psychological stress and had frequent bouts of confirmed abdominal angioedema (Fig. 8). The majority of icatibant-treated attacks $(68.4 \%$; $39 / 57)$ were abdominal. The patient was receiving long-term danazol prophylaxis at attack onset for $49.1 \%$ (28/57) of icatibant-treated attacks. However, in spring 2011 prophylaxis with danazol became contraindicated owing to the development of abnormal liver function tests, hyperlipidaemia and weight gain. The patient initiated long-term prophylaxis with C1-INH in April 2011 and has reported only three icatibant-treated HAE attacks since that date.

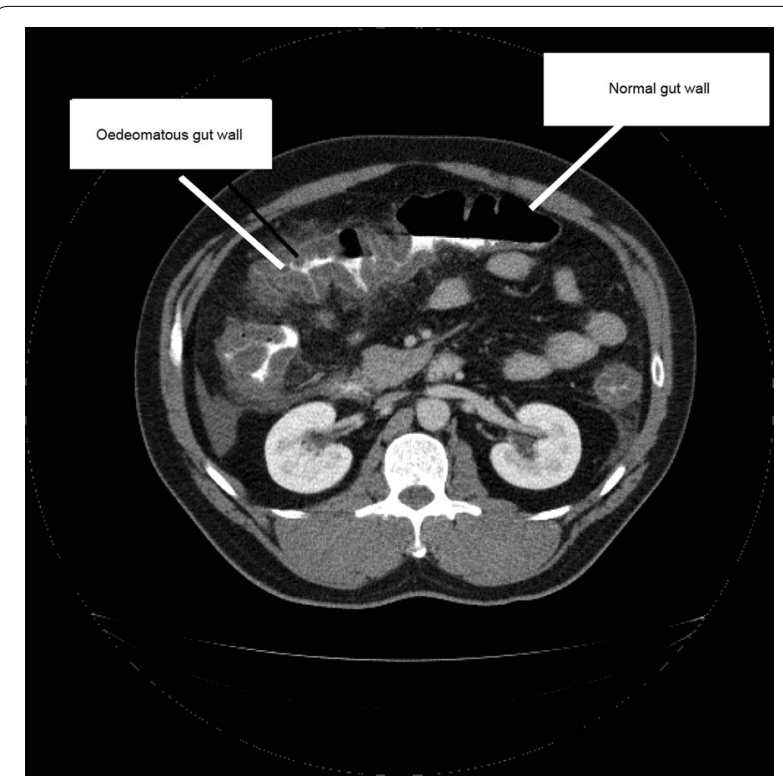

Fig. 8 Abdominal computed tomography image obtained at admission of a 47-year-old male patient with HAE type I with abdominal angioedema 
Table 5 UK patients with acquired angioedema

\begin{tabular}{llllll}
\hline Patient no. & Gender & $\begin{array}{l}\text { Age at IOS enrolment, } \\
\text { years }\end{array}$ & $\begin{array}{l}\text { Icatibant-treated angi- } \\
\text { oedema attacks } \mathbf{a}^{\mathbf{n}}\end{array}$ & $\begin{array}{l}\text { HCP-administered icatibant } \\
\text { injections, } \boldsymbol{n}\end{array}$ & $\begin{array}{l}\text { Self-administered } \\
\text { icatibant injections, } \boldsymbol{n}\end{array}$ \\
\hline 1 & Male & 50.5 & 53 & 3 & 51 \\
2 & Male & 63.1 & 27 & 6 & 22 \\
3 & Female & 49.7 & 2 & 1 & 1 \\
4 & Male & 70.9 & 1 & - & 1 \\
5 & Female & 28.6 & 0 & - & - \\
6 & Female & 59.8 & 0 & - & - \\
7 & Female & 38.4 & 0 & - & - \\
\hline
\end{tabular}

HCP healthcare professional, IOS Icatibant Outcome Survey

a July 2009 to July 2016

\section{Acquired angioedema}

Seven patients from the UK and 34 non-UK patients had a diagnosis of AAE due to C1-INH deficiency. Icatibant is not currently licensed for use in patients with AAE due to C1-INH deficiency and these patients were not included in the analysis described above. However, for completeness of the UK dataset, we include a brief description of the seven UK patients in Table 5 [23].

\section{Discussion}

IOS has allowed the follow-up of patients with HAE type I/II in the UK and centres across Europe, the Middle East and South America. These data from three UK centres suggest that HAE remains under-recognized in the UK, as shown by long diagnostic delays. Whilst some patients were diagnosed prior to first symptoms (presumably based on family history) or at the time of or soon after first symptoms, others experienced very long delays, with consequent increased risk of avoidable mortality and morbidity. The delay in diagnosis in the UK $(n=73)$ was comparable to that in non-UK countries, which supersedes the previously reported trend from a smaller IOS UK patient population $(n=12)$ [24]. The delay in diagnosis in the UK was shorter than the delay in diagnosis recently reported in a UK National Audit of HAE; however this may be explained by differences in methodology, as the authors of the UK National Audit excluded all patients who received diagnoses before experiencing symptoms [20]. The overarching message from all these studies is that delay in diagnosis is highly variable; a recent IOS analysis found approximately $50 \%$ of patients with HAE type I/II had received a prior misdiagnosis, most commonly allergic angioedema or appendicitis [25]. Increased awareness of HAE could reduce time to diagnosis and permit appropriate management of the condition, thus reducing the morbidity and mortality associated with undiagnosed HAE [26].
Self-administration of HAE treatment at home offers the potential for earlier treatment and symptom control [21], reducing the impact of HAE on physical, social and economic well-being and reducing healthcare resource use $[8,27,28]$. The UK patients included a higher proportion of attacks treated by self-administration compared with non-UK patients $(95.8 \%$ versus $86.8 \%$, respectively). This may partially be due to the later enrolment of patients in the UK, with 66/73 (90.4\%) patients joining the study in 2011 or later, compared with a larger number of patients in Europe (177/579 [30.6\%]) who enrolled up to 2 years prior to the 2011 change in indication to include self-administration. Importantly, each of our UK centres in IOS is a strong advocate of home therapy and patient empowerment, in accordance with UK government policy and home therapy guidance $[3,7,10]$. There is long-standing experience of treating and training patients for self-administration in the UK, compared with more recent experience in some other European countries $[15,29]$. This is greatly facilitated by the availability of immunology specialist nurses who develop considerable expertise in self-administration training and patient support [14, 29, 30]. Although a higher proportion of UK patients self-administered icatibant compared with non-UK patients, the proportion of first attacks treated by self-administration was similar for the UK sample and non-UK patients (71.9\% versus $63.7 \%$, respectively). It is recommended that the first dose of icatibant is given under the supervision of a healthcare professional to ensure adequate training [12] and allow monitoring of tolerability to the drug immediately after administration. However, the need for professional supervision can be a barrier to accessing treatment and therefore remains a recommendation rather than a requirement. These IOS data support our clinical experience that patients tolerate icatibant well and, with encouragement and support, rarely require more than one training session. Moreover, 
the ease of use of icatibant has enabled a greater proportion of patients to self-administer treatment for $\mathrm{HAE}$ attacks than is possible with $\mathrm{C} 1-\mathrm{INH}$ treatment, which requires intravenous access.

Time to treatment, time to resolution and duration of attack were not significantly different in the UK compared with non-UK patients. Previously, using a data extract of April 2015 (presented at UK PIN meeting 2015), we had reported that UK IOS patients experience a significantly shorter time to treatment, time to resolution and attack duration than patients from other IOS countries. However, the July 2016 data presented herein clearly show this gap has closed, and a recent country comparison of IOS data provides additional information regarding regional variations in both delay in diagnosis and icatibant use across six EU member states [31]. Previous IOS analyses have demonstrated that earlier treatment can reduce both overall attack duration and time to resolution [21].

Patients with HAE suffer impaired quality of life and incapacity, with time away from school, work, social events and family life, and reduced productivity [2, 32-39]. A higher proportion of the UK patients were employed or in education compared with non-UK patients. In the UK, a higher percentage $(72.6 \%)$ were employed or self-employed compared with the UK national audit of HAE (48\% in paid employment) [20]. As IOS is an icatibant registry, it may be that factors that lead to icatibant prescription result in a degree of selection bias, thereby giving rise to the differences between this population and the wider group of UK patients with HAE. However, the data could also be consistent with the early adoption of icatibant by the specialist centres studied and the inclusion of data on patients after 2013, when barriers to icatibant prescription were relaxed in the UK following adoption of a policy of central funding [8]. Patients in the UK missed more time from work owing to HAE than non-UK patients. Despite needing to miss more work for HAE attacks, a higher percentage of UK patients were able to maintain employment, perhaps because more patients self-treated with icatibant. This suggests that an effective self-administered treatment for angioedema attacks may be an important element in enabling patients to stay in work [8]. For example, one of the patients with AAE due to C1-INH deficiency had experienced significant employment difficulties that were resolved once they had access to icatibant at home and at work. The proportion of patients with no HAE-related time off work improved after inclusion in IOS. However, there was no improvement in those with high levels ( $>7$ days per annum) of HAE-related work absenteeism, suggesting that additional measures are required for this group.
Patients in IOS were managed according to routine clinical practice, providing a comprehensive and an accurate 'real-world' description of HAE patients with C1-INH deficiency at three major NHS-funded specialist centres. This prospective registry included only patients who were prescribed icatibant. Factors influencing icatibant prescription have not been formally characterized, but may include previous treatment experience and training, quality of venous access, attack frequency, patient preference, religious beliefs (concerning blood products) and funding decisions (HJ Longhurst, personal communication). These factors may have an impact on the generalizability of the results. In addition, UK data were geographically limited to three large, specialist HAE centres in London, Manchester and Plymouth; therefore, referral bias is inevitable, and data may not be representative of the wider UK experience (UK patients represented approximately $3.5 \%$ of the UK HAE population). We believe that continued national commissioning will reduce inequalities of access to treatment and may allow wider access to icatibant in the future.

Other limitations of the analyses, common to most observational studies, include: only patients with available data are analysed; missing data for some endpoints; the variable length of patient follow-up; and potential variability of data collection methodology for attacks occurring prior to IOS entry and during the IOS observational period. In addition, attack severity was described by the patients, therefore, may have been subjective, and a new attack or a relapse of a previous attack were indistinguishable. Furthermore, patients who self-administer icatibant treatment may not follow recommended practice, despite receiving training, and the response to icatibant may vary according to patient practice. The case we described of a patient with very high reinjection and C1-INH rescue use demonstrates that patients' may develop different treatment practices and highlights that physicians should carefully monitor patients' use of treatment. However, occasional use of icatibant as a 'holding' treatment in patients who routinely use C1-INH for very frequent attacks can, in our experience, be useful in immediately life-threatening situations or where access to $\mathrm{C} 1-\mathrm{INH}$ is likely to be subject to unacceptable delay.

\section{Conclusions}

Data from IOS have helped characterize the experience of HAE patients at three UK centres. Our findings suggest that availability of icatibant treatment may have encouraged patients to become more involved in their care through self-administration, with improvement in some outcomes such as increased rate of treatment for potentially debilitating attacks and HAE-related time off work or study. Compared with their non-UK peers, a 
higher proportion of UK patients were in work or studying. Those using icatibant reported less time off work than their non-UK counterparts. Ultimately, we believe that HAE management in the UK should focus on the maintenance of wider patient social and physical functioning. Improvements are required to further reduce the unacceptable diagnostic delay and to address the problem of work absenteeism in those requiring more than 7 days off work. The latter may require a 'whole of life' approach to disability prevention.

We anticipate publishing a UK IOS update every 3-5 years to help establish benchmarking standards and inform improvements to the health and quality of life in individuals with C1-INH deficiency.

\section{Abbreviations \\ AAE: acquired angioedema; BL: baseline; $\mathrm{C1}$-INH: $\mathrm{C} 1$ inhibitor; HAE: hereditary angioedema; HCP: healthcare professional; IOS: Icatibant Outcome Survey;} IQR: interquartile range; SD: standard deviation.

\section{Authors' contributions}

HJL, JD, LL, SG, MB, CB, CS, CBe, TG all contributed to study conception and design; data acquisition, analysis and interpretation; drafting of the manuscript and critical content revisions; and final approval of content. VF contributed to the study conception and design; analysis and interpretation; drafting of the manuscript and critical content revisions and final approval of content. All authors read and approved the final manuscript.

\section{Author details}

${ }^{1}$ Department of Clinical Biochemistry and Immunology, Addenbrooke's Hospital, Cambridge University Hospitals NHS Foundation Trust, Cambridge, UK. ${ }^{2}$ Department of Immunology, Barts Health NHS Trust, London, UK. ${ }^{3}$ Department of Immunology, Plymouth Hospitals NHS Trust, Plymouth, UK. ${ }^{4}$ Shire, Zug, Switzerland. ${ }^{5}$ Department of Immunology, Central Manchester University Hospital NHS Foundation Trust, Manchester, UK.

\section{Acknowledgements}

Medical writing support was provided by Tracey Spurway Ph.D. of Complete Medical Communications, Macclesfield, UK, and David Lickorish Ph.D., CMPP, of Excel Scientific Solutions, Southport, CT, and was funded by Shire Human Genetic Therapies, Lexington, MA, USA.

The following IOS investigators and study staff are also acknowledged for their contributions to the IOS

Austria:W. Aberer; Brazil: A. S. Grumach; Denmark: A. Bygum; France: C. Blanchard Delaunay, I. Boccon-Gibod; L. Bouillet, B. Coppere, A. Du Thanh, C. Dzviga, O. Fain, B. Goichot, A. Gompel, S. Guez, P. Y. Jeandel, G. Kanny, D. Launay, H. Maillard, L. Martin, A. Masseau, Y. Ollivier, A. Sobel; Germany: E. AygörenPürsün, M. Bas, M. Bauer, K. Bork, J. Greve, M. Magerl, I. Martinez-Saguer, M. Maurer, U. Strassen; Greece: E. Papadopoulou-Alataki, F. Psarros; Israel:Y. Graif, S. Kivity, A. Reshef, E. Toubi; Italy: F. Arcoleo, M. Bova, M. Cicardi, P. Manconi, G. Marone, V. Montinaro, M. Triggiani, A. Zanichelli; Spain: M. L. Baeza, T. Caballero, R. Cabanas, M. Guilarte, D. Hernandez, C. Hernando de Larramendi, R. Lleonart, T. Lobera, L. Marques, B. Saenz de San Pedro; Sweden: J. Björkander; UK: C. Bethune, T. Garcez, H. J. Longhurst.

\section{The IOS Executive Committee}

W. Aberer, L. Bouillet, A. Bygum, T. Caballero, A. S. Grumach, H. J. Longhurst, M. Maurer, A. Zanichelli.

\section{Competing interests}

HJL has received research grant support and/or speaker/consultancy fees from BioCryst, CSL Behring, Dyax, Shire and Sobi. JD has acted as a consultant/ conducted research for CSL Behring, Shire and SOBI. LL has received research funding from Shire and conducted research for CSL Behring and Shire. MB has acted as a consultant for Shire and received support for meeting attendance from Shire. SG has received support for meeting attendance from CSL Behring and Shire. CS has received support for meeting attendance from Shire and CSL Behring. CBe has received support for meeting attendance and speaker/ consultancy fees from CSL Behring and Shire. VF was an employee of Shire, Zug, Switzerland during the conduct of the study and development of the manuscript. CB has no conflicts of interest to declare. TG has received support for meeting attendance from CSL Behring and Shire, has conducted research for Biocryst and Shire and contributes to advisory boards for CSL Behring and Baxalta/Shire.

\section{Availability of data and materials}

The datasets generated during the current study are not publicly available because the data from IOS reside in a proprietary database maintained by Shire but data are available from the corresponding author on reasonable request and with permission of Shire.

\section{Ethics approval and consent to participate}

IOS is conducted in accordance with the Declaration of Helsinki and the International Conference on Harmonisation Good Clinical Practice. Approval was obtained from ethics committees and/or local health authorities at all centres. Written, informed consent was obtained from all enrolled patients aged $\geq 18$ years ( $>16$ years in the UK); consent was provided by parents/legal guardians for patients aged $<18$ years $(<16$ years in the UK).

\section{Funding}

IOS is funded and supported by Shire International GmbH, Zug, Switzerland.

\section{Previous presentation}

Data from an April 2015 IOS data extract were presented in a poster at the UK Primary Immunodeficiency Network Annual Meeting, 19-20 November 2015, Belfast, UK. Data in the present manuscript (July 2016 data extract) have not been presented previously in any form.

\section{Publisher's Note}

Springer Nature remains neutral with regard to jurisdictional claims in published maps and institutional affiliations.

Received: 24 August 2017 Accepted: 9 April 2018

Published online: 06 August 2018

\section{References}

1. Cicardi M, Johnston DT. Hereditary and acquired complement component 1 esterase inhibitor deficiency: a review for the hematologist. Acta Haematol. 2012;127:208-20.

2. Longhurst $\mathrm{H}$, Cicardi M. Hereditary angio-oedema. Lancet. 2012;379:474-81.

3. Manson AL, Price A, Dempster J, Clinton-Tarestad P, Greening C, Enti R, et al. In pursuit of excellence: an integrated care pathway for $\mathrm{C} 1$ inhibitor deficiency. Clin Exp Immunol. 2013;173:1-7.

4. Bowen T, Cicardi M, Farkas H, Bork K, Longhurst HJ, Zuraw B, et al. 2010 International consensus algorithm for the diagnosis, therapy and management of hereditary angioedema. Allergy Asthma Clin Immunol. 2010;6:24.

5. Cicardi M, Bork K, Caballero T, Craig T, Li HH, Longhurst H, et al. Evidencebased recommendations for the therapeutic management of angioedema owing to hereditary C1 inhibitor deficiency: consensus report of an international working group. Allergy. 2012;67:147-57.

6. Craig T, Aygören-Pürsün E, Bork K, Bowen T, Boysen H, Farkas H, et al. WAO guideline for the management of hereditary angioedema. World Allergy Organ J. 2012;5:182-99.

7. Gompels MM, Lock RJ, Abinun M, Bethune CA, Davies G, Grattan C, et al. C1 inhibitor deficiency: consensus document. Clin Exp Immunol. 2005;139:379-94

8. Longhurst HJ, Farkas H, Craig T, Aygören-Pürsün E, Bethune C, Bjorkander $J$, et al. HAE international home therapy consensus document. Allergy Asthma Clin Immunol. 2010;6:22. 
9. Longhurst HJ, Tarzi MD, Ashworth F, Bethune C, Cale C, Dempster J, et al. C1 inhibitor deficiency: 2014 United Kingdom consensus document. Clin Exp Immunol. 2015;180:475-83.

10. NHS commissioning board clinical commissioning policy: treatment of acute attacks in hereditary angioedema. NHS commissioning board; 2013. https://www.england.nhs.uk/commissioning/wp-content/uploads/ sites/12/2013/09/b09-p-b.pdf. Accessed 13 Apr 2017.

11. Berinert $500 \mathrm{IU}$ (summary of product characteristics). CSL Behring; 2013.https://www.medicines.org.uk/emc/medicine/21650. Accessed 13 Apr 2017

12. Firazyr $30 \mathrm{mg}$ solution for injection in pre-filled syringe (summary of product characteristics). Shire; 2013. https://www.medicines.org.uk/ emc/medicine/21761/SPC/Firazyr+30+mg+solution+for+injec tion+in+pre-filled+syringe/. Accessed 13 Apr 2017.

13. Cinryze 500 units powder and solvent for solution for injection (summary of product characteristics). Shire; 2012. https://www.medicines.org.uk/ emc/medicine/25634. Accessed 13 Apr 2017.

14. Aberer W, Maurer M, Reshef A, Longhurst H, Kivity S, Bygum A, et al. Open-label, multicenter study of self-administered icatibant for attacks of hereditary angioedema. Allergy. 2013;69:305-14.

15. Fernandez Hernández, de Rojas $D$, Ibañez E, Longhurst $H$, Maurer $M$, Fabien V, Aberer W, et al. Treatment of HAE Attacks in the icatibant outcome survey: an analysis of icatibant self-administration versus administration by health care professionals. Int Arch Allergy Immunol. 2015;167:21-8.

16. Bygum $\mathrm{A}$. Hereditary angio-oedema in Denmark: a nationwide survey. $\mathrm{Br}$ J Dermatol. 2009;161:1153-8.

17. Grumach AS, Valle SO, Toledo E, de Moraes Vasconcelos D, Villela MM,

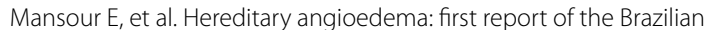
registry and challenges. J Eur Acad Dermatol Venereol. 2013;27:e338-44.

18. Mallbris L, Nordenfelt P, Björkander J, Lindfors A, Werner S, Wahlgren CF. The establishment and utility of Sweha-Reg: a Swedish population-based registry to understand hereditary angioedema. BMC Dermatol. 2007;7:6.

19. Roche O, Blanch A, Caballero T, Sastre N, Callejo D, López-Trascasa M. Hereditary angioedema due to $C 1$ inhibitor deficiency: patient registry and approach to the prevalence in Spain. Ann Allergy Asthma Immunol. 2005:94:498-503.

20. Jolles S, Williams P, Carne E, Mian H, Huissoon A, Wong G, et al. A UK national audit of hereditary and acquired angioedema. Clin Exp Immunol. 2014;175:59-67.

21. Maurer M, Aberer W, Bouillet L, Caballero T, Fabien V, Kanny G, et al. Hereditary angioedema attacks resolve faster and are shorter after early icatibant treatment. PLoS ONE. 2013;8:e53773.

22. Cicardi M, Banerii A, Bracho F, Malbrán A, Rosenkranz B, Riedl M, et al. Icatibant, a new bradykinin-receptor antagonist, in hereditary angioedema. N Engl J Med. 2010:363:532-41.

23. Longhurst HJ, Zanichelli A, Caballero T, Bouillet L, Aberer W, Maurer M, et al. Comparing acquired angioedema with hereditary angioedema (types I/II): findings from the icatibant outcome survey. Clin Exp Immunol. 2016;188(1):148-53

24. Zanichelli A, Magerl M, Longhurst H, Fabien V, Maurer M. Hereditary angioedema with $\mathrm{C} 1$ inhibitor deficiency: delay in diagnosis in Europe. Allergy Asthma Clin Immunol. 2013:9:29.
25. Zanichelli A, Longhurst HJ, Maurer M, Bouillet L, Aberer W, Fabien V, et al. Misdiagnosis trends in patients with hereditary angioedema from the real-world clinical setting. Ann Allergy Asthma Immunol. 2016;1 17:394-8.

26. Bork K, Hardt J, Witzke G. Fatal laryngeal attacks and mortality in hereditary angioedema due to C1-INH deficiency. J Allergy Clin Immunol. 2012;130:692-7.

27. Bygum A, Andersen KE, Mikkelsen CS. Self-administration of intravenous C1-inhibitor therapy for hereditary angioedema and associated quality of life benefits. Eur J Dermatol. 2009;19:147-51.

28. Caballero T, Sala-Cunill A, Cancian M, Craig TJ, Neri S, Keith PK, et al. Current status of implementation of self-administration training in various regions of Europe, Canada and the USA in the management of hereditary angioedema. Int Arch Allergy Immunol. 2013;161(Suppl 1):10-6.

29. Symons C, Rossi O, Magerl M, Andritschke K. Practical approach to selfadministration of intravenous C1-INH concentrate: a nursing perspective. Int Arch Allergy Immunol. 2013;161(Suppl 1):17-20.

30. Dempster J. Management of hereditary angioedema. Nurs Stand. 2013;27:35-40.

31. Caballero T, Aberer W, Longhurst HJ, Maurer M, Zanichelli A, Perrin A, et al. The icatibant outcome survey: experience of hereditary angioedema management from six European countries. J Eur Acad Dermatol Venereol. 2017:31(7):1214-22

32. Banerji A. The burden of illness in patients with hereditary angioedema. Ann Allergy Asthma Immunol. 2013;111:329-36.

33. Bowen T. Hereditary angioedema: beyond international consensuscirca December 2010-the Canadian Society of allergy and clinical immunology Dr. David McCourtie Lecture. Allergy Asthma Clin Immunol. 2011;7:1.

34. Lumry WR, Castaldo AJ, Vernon MK, Blaustein MB, Wilson DA, Horn PT. The humanistic burden of hereditary angioedema: impact on healthrelated quality of life, productivity, and depression. Allergy Asthma Proc. 2010;31:407-14.

35. Wilson DA, Bork K, Shea EP, Rentz AM, Blaustein MB, Pullman WE. Economic costs associated with acute attacks and long-term management of hereditary angioedema. Ann Allergy Asthma Immunol. 2010;104:314-20

36. Caballero T, Aygören-Pürsün E, Bygum A, Beusterien K, Hautamaki E, Sisic $Z$, et al. The humanistic burden of hereditary angioedema: results from the burden of illness study in Europe. Allergy Asthma Proc. 2014:35:47-53.

37. Bygum A, Aygören-Pürsün E, Beusterien $K$, Hautamaki E, Sisic Z, Wait S, et al. Burden of illness in hereditary angioedema: a conceptual model. Acta Derm Venereol. 2014;95(6):706-10.

38. Nordenfelt P, Dawson S, Wahlgren CF, Lindfors A, Mallbris L, Björkander J. Quantifying the burden of disease and perceived health state in patients with hereditary angioedema in Sweden. Allergy Asthma Proc. 2014;35:185-90.

39. Longhurst $\mathrm{H}$, Bygum A. The humanistic, societal, and pharmaco-economic burden of angioedema. Clin Rev Allergy Immunol. 2016;51:230-9.

Ready to submit your research? Choose BMC and benefit from

- fast, convenient online submission

- thorough peer review by experienced researchers in your field

- rapid publication on acceptance

- support for research data, including large and complex data types

- gold Open Access which fosters wider collaboration and increased citations

- maximum visibility for your research: over $100 \mathrm{M}$ website views per year

At BMC, research is always in progress.

Learn more biomedcentral.com/submissions 